is to look through a filter of density 4.5 - "This means that a filter should not transmit more than one part in 30,000 of the total solar radiation." But who knows whether a filter, especially if improvised, has the right density ? In fact the best way for the amateur observer to see an eclipse is by projecting its image through a pin-hole on to a sheet of card or paper, ${ }^{45}$ which may be black ${ }^{1}$ or white. ${ }^{6}$

\section{Venous Ulcers}

The treatment of varicose veins and their complications is always a matter of controversy, and though it is a good sign that such a common condition ${ }^{1}$ can stimulate prolonged and heated activity in our correspondence columns it is also indicative of our ignorance of the basic abnormalities and that our treatment is often illogical and empirical. No one denies that the primary cause of venous (or varicose, or orthostatic-take your pick) ulcers is the venous hypertension which develops in incompetent veins during erect exercise, yet how this abnormality produces the induration, pigmentation, fat necrosis, eczema, and ulceration has not been satisfactorily explained. Numerous hypotheses have been advanced. The oedema has been blamed on changes of capillary permeability ${ }^{2}$ - a most difficult thing to measure in intact man. Changes in tissue nutrition have been blamed on the presence of arteriovenous fistulae, ${ }^{3-5}$ a suggestion that could also explain the increased oxygen tension of the blood draining the region of a venous ulcer. Capillary fragility with interstitial haemorrhage, fat necrosis, and ultimately ulceration may be due to either direct trauma from outside or indirect trauma from the internal activity of the muscles and the rapid changes of venous pressure. With so many possible aetiological factors it is not surprising that a multitude of remedies are in use, nor that the only ones that are really effective are those that control the venous hypertension-for this must be the prime cause. Thus the closure of the incompetent superficial-to-deep connecting veins by surgery ${ }^{6}$ or sclerotherapy ${ }^{7}$ will produce a permanent cure in most cases. Alternatively, simple elevation or lateral counter-pressure with elastic stockings, adhesive strapping, or impregnated bandages will produce a temporary cure, and such treatment is often necessary as an accompaniment or preliminary to the definitive treatment of the incompetent veins.

It is also a fair comment that any form of treatment aimed at the secondary effects of the venous hypertension is unlikely to be curative and may not help at all. Diuretics are of little value. Local or systemic antibiotics are not indicated, for most varicose ulcers are not infected with virulent pathogens -another interesting and unexplained feature. The medicaments in the impregnated bandages, such as zinc oxide and ichthyol, probably do no more than make the bandage stiffer and harder and consequently a better support. Interest has recently shifted towards the changes in capillary permeability, and substances such as troxerutin, which have been shown to affect capillary permeability, ${ }^{8}$ are claimed to be of value. The problems of assessing such drugs in the absence of an acceptable method of measuring capillary permeability in the margins of a venous ulcer in man are extremely difficult. Those studies so far published suggest that this type of approach will yield only small fringe benefits. ${ }^{9}$
The problem always comes back to the major known abnormality-venous hypertension-and unless this is abolished a permanent cure cannot be achieved. Yet this is more easily said than done and requires a painstaking clinical examination and repeated follow-up, for quite frequently the first assault misses some of the abnormal perforating vessels. The vast number of patients affected makes the use of special techniques such as phlebography or pressure studies impractical, and even these techniques are not infallible. So these patients must be treated on the basis of clinical examination, and because they may require more than one operation the relatively inexpensive injection techniques revived by G. W. Fegan ${ }^{7}$ are appealing. What matters most is that the whole problem of varicose veins and venous ulceration should be approached in an exact and scientific manner: the ability to produce rapid relief from the symptoms of this disease is of great economic importance.

\section{Cheltenham Meeting}

The Annual Clinical Meeting of the British Medical Association at Cheltenham next month is to be a joint one with the British Paediatric Association. The B.M.A.'s practice, which began last year, of inviting a specialist society to join in its clinical meetings is a good one. Since the division of the Annual Scientific Meeting into specialist sections was discontinued some seven years ago there has been a danger that the B.M.A. might be thought to have less concern for specialist medicine. The new practice is a valuable corrective to any such trend, and the Association's Board of Science, under the chairmanship of Professor Sir Ronald Tunbridge, may be expected to continue it.

The emphasis in the programme for the Cheltenham Meeting, printed in the Supplement, is on subjects of interest to the practising doctor. Among them are virus diseases, allergic reactions in the lungs, malabsorption syndromes, and a variety of special topics in paediatric medicine. The sessions are recognized for the purposes of the postgraduate training allowance for N.H.S. general practitioners. Lord Todd is to give the opening address entitled "The Doctor in the Changing World."

A more pleasant venue for a meeting than Regency Cheltenham, together with its beautiful surrounding country, could scarcely be imagined, and there is an attractive programme of visits to nearby places of interest. The Meeting coincides with the centenary of the Gloucestershire Branch of the B.M.A., and visitors will have the privilege of being able to join in a service of thanksgiving in Gloucester Cathedral on 27 October.

\footnotetext{
Borschberg, E., The Prevalence of Varicose Veins in the Lower Extremities, 1968. Basel.

2 Myers, T. T. in Peripheral Vascular Diseases, 1962, 3rd edition. ed. E. V. Allen, N. W. Barker, and E. A. Hines. London.

3 King, E. S. J., Aust. N.Z. \}. Surg., 1950, 20, 126.

- Piulachs, P., and Vidal-Barraquer, F., Angiology, 1953, 4, 59.

s Gius, J. A., Arch. Surg., 1960, 81, 299.

6 Dodd, H., and Cockett, F. B., The Pathology and Surgery of the Veins of the Lower Limb, 1956. London.

- Fegan, G. W., Varicose Veins, 1967. London.

- Wismer, R., Praxis, 1963, 52, 1412.

- Fitzgerald, D. E., Practitioner, 1967, 198, 406.
} 